\title{
Summation in autoshaping
}

\author{
ROBERT A. RESCORLA and SUSAN E. COLDWELL \\ University of Pennsylvania, Philadelphia, Pennsylvania
}

\begin{abstract}
Pigeon subjects received Pavlovian conditioning with stimulus elements and were then tested with compounds of those elements. Experiments 1-3 used localized keylight elements and found no evidence for greater responding to the compound than to the elements. Experiments 4A-4D found evidence for greater second-order conditioning by a compound of two elements than by the elements themselves when the elements consisted of two diffuse stimuli or one diffuse stimulus and one localized keylight. No greater second-order conditioning resulted from a compound of two localized keylight elements, suggesting the possibility of perceptual interactions that reduce identification of the elements in the compound. Experiment 6 found evidence of summation when that interaction was reduced by sequential presentation. However, one attempt to capture this interaction in terms of configural conditioning (Pearce, 1987) failed to receive confirmation. These results suggest that the localized stimuli conventionally employed in autoshaping experiments may show such substantial perceptual interaction as to recommend against their routine use for studying conditioning in compounds.
\end{abstract}

Many modern experiments on associative learning involve the presentation of complex stimuli composed of separable elements. For this reason, it has been of continuing interest to understand the relation between the associative strength of a compound stimulus and that of the elements of which it is composed. Perhaps the simplest experiment addressing that question is one in which responding is assessed to a compound after its elements have been separately trained. Beginning with Pavlov (1927), many authors have reported a result labeled as summation (i.e., greater response to the compound than to either of the elements alone). Although that summation is rarely linear in form, it has been reported for a wide variety of learning preparations. For instance, summation apparently occurs in such Pavlovian paradigms as eyelid conditioning (e.g., Kehoe, 1986; Whitlow \& Wagner, 1972), conditioned suppression (e.g., Reberg, 1972), flavor aversion (e.g., Forbes \& Holland, 1985), magazine entry (e.g., Bellingham, Gillette-Bellingham, \& Kehoe, 1985 ), and salivary conditioning. Similarly, summation occurs in both appetitive and aversive instrumental learning preparations (Weiss, 1972).

Partly as a result of such observations, many historically important theories of conditioning have included some explicit combination rule by which the associative strength of a stimulus compound is an additive function of the strength of its elements (e.g., Hull, 1943; Mackintosh, 1975; Pearce \& Hall, 1980; Rescorla \& Wagner, 1972). More recent network theories have also assumed that elements of a com-

This research was supported by National Science Foundation Grant BNS-88-03514. Correspondence concerning this article should be addressed to Robert A. Rescorla, Department of Psychology, University of Pennsylvania, 3815 Walnut Street, Philadelphia, PA 19104.

-Accepted by previous editor, Vincent M. LoLordo pound summate in order to generate the associative strength (e.g., Rummelhart \& McClelland, 1986).

But there is reason to doubt that such summation routinely occurs in one popular conditioning preparation, autoshaping with pigeons. In that preparation, a localized visual stimulus signals the availability of grain. As a result of learning this relation, the bird directs pecking at the stimulus. However, we are aware of no reports explicitly demonstrating summation in that preparation. Moreover, there are various results that suggest that the concurrent presentation of two such stimuli may not generate a summated pecking rate. For instance, Rescorla (1989) observed only a marginally higher response rate to a green horizontal line compound when each element was separately reinforced. Similarly, Pearce, Adam, Wilson, and Darby (1992) tested various threeelement compounds after conditioning the components as elements or as two-element compounds. Although their interest was in differences among the triplets, comparison between responding to the elements at the end of training and responding to the triplets in the test suggests that no summation was observed.

These failures to observe summation are of particular interest because they may be consistent with an approach that views compound stimuli as configurations different from the sum of the component parts. Although elementaristic theories of conditioning have been historically dominant, configural theories have continued to be suggested. Of course, one difficulty faced by this sort of theory is to specify the relation among compounds and between compounds and their component parts. Since training of two stimulus elements results in some level of responding when they are presented in compound, it is apparent that organisms do not treat the compound as an entirely new event unrelated to its elements. One recent attempt to specify their relation 
within a configural framework that has enjoyed considerable empirical success has been introduced by Pearce (1987). According to this view, an AB compound, composed by the experimenter of $A$ and $B$, is treated by the animal as a separate stimulus that gains its own associative strength different from that of $A$ and $B$. However, there is also generalization among $\mathrm{A}, \mathrm{B}$, and $\mathrm{AB}$, depending on the degree to which they share features. In summation experiments, $A B$ is not itself trained; hence, this generalization to $\mathrm{AB}$ from the $\mathrm{A}$ and $\mathrm{B}$ elements entirely accounts for performance to the $\mathrm{AB}$ compound. According to this configural model, that generalization depends on the similarity $(S)$ of $\mathrm{A}$ and $\mathrm{B}$ to $\mathrm{AB}$, which is specified in terms of the intensity $(I)$ of the elements two stimuli share. Put in the notation of that theory, the similarity of $\mathrm{A}$ to $\mathrm{AB},{ }_{\mathrm{A}} S_{\mathrm{AB}}$, is equal to the proportion of the total intensity of the $\mathrm{AB}$ compound $\left(I_{\mathrm{A}}+I_{\mathrm{B}}\right)$ that resides in the shared A component $\left(I_{\mathrm{A}}\right)$ [i.e., $\left.I_{\mathrm{A}} /\left(I_{\mathrm{A}}+I_{\mathrm{B}}\right)\right]$. Hence, the associative strength of the $A B$ compound after conditioning of $A$ and $B$ is equal to the generalization it gets from each of $\mathrm{A}$ and $\mathrm{B}$. If $V_{\mathrm{i}}$ is the associative strength of stimulus $\mathrm{i}$, then $V_{\mathrm{AB}}$ equals $V_{\mathrm{A}} \times{ }_{\mathrm{A}} S_{\mathrm{AB}}+V_{\mathrm{B}}$ $\times{ }_{\mathrm{B}} S_{\mathrm{AB}}$. Given the definition of $S_{\mathrm{AB}}$, this means that $V_{\mathrm{AB}}$ is a weighted average of $V_{\mathrm{A}}$ and $V_{\mathrm{B}}$ [i.e., $V_{\mathrm{AB}}=V_{\mathrm{A}} \times$ $\left.\left(I_{\mathrm{A}}\right) /\left(I_{\mathrm{A}}+I_{\mathrm{B}}\right)+V_{\mathrm{B}} \times\left(I_{\mathrm{B}}\right) /\left(I_{\mathrm{A}}+I_{\mathrm{B}}\right)\right]$. In the simplest case where $V_{\mathrm{A}}$ and $V_{\mathrm{B}}$ are equal and have similar saliences, this expression reduces to $V_{\mathrm{A}}\left(=V_{\mathrm{B}}\right)$. That is, this specification of a configural model anticipates that responding to $A B$ will in general be the same as that to $A$ and $B$ separately (i.e., that no summation will occur). This quite clearly contrasts with the predictions of most elemental theories.

The present experiments therefore sought to explore more thoroughly the degree to which such summation occurs in autoshaping, a conditioning paradigm frequently used to choose among such alternative theories. Experiment 1 was a straightforward comparison of responding to each of two trained visual stimuli and to their tested compound. Because no evidence of summation was found in Experiment 1, Experiments 2, 3, and 4 explored potential performance factors that might mask underlying associative summation. They tested various different types of stimuli and included a measure of conditioning other than the ability of the compound to elicit responding - namely, second-order conditioning. The results of these experiments suggest that there can be a perceptual interaction between localized visual stimuli that results in the compound's failing to preserve fully the integrity of the elements. Experiment 5 evaluated whether the configural model proposed by Pearce could successfully characterize this interaction. Experiment 6 explored an empirical procedure that might be expected to reduce that interaction and, hence, produce summation.

\section{EXPERIMENT 1}

In Experiment 1, animals received simultaneous compound presentations of stimuli that had different conditioning histories. Initially, they were taught two discrim- inations of the form $\mathrm{A}+/ \mathrm{B}-$ and $\mathrm{C}+/ \mathrm{D}-$, where all four stimuli were localized keylights. Then they were tested with $\mathrm{C}$ and $\mathrm{D}$ alone and in combination with $\mathrm{A}$ and $\mathrm{B}$. The issue was whether responding to the $C$ compounded with the excitatory $\mathrm{A}$ will be greater than that to $\mathrm{C}$ alone and to $\mathrm{C}$ compounded with the nonreinforced $\mathrm{B}$.

\section{Method}

Subjects and Apparatus. The subjects were 16 female Carneau pigeons, each about 1 year old. They had participated in another autoshaping experiment that had used different stimuli and a different response key. The assignment of birds to groups in the present experiment was random with respect to their previous treatments. They were housed in pairs and maintained at $80 \%$ of their free-feeding weights.

The apparatus consisted of eight identical operant chambers, each measuring $27 \times 27 \times 35 \mathrm{~cm}$. The metal front panel of each chamber had a $5 \times 5 \mathrm{~cm}$ food magazine in its center, located $5 \mathrm{~cm}$ above the wire mesh floor. An $11.8 \times 14.5 \mathrm{~cm}$ response key, constructed of clear Lucite acrylic, was centered $9 \mathrm{~cm}$ above the magazine, behind a $10 \times 8 \mathrm{~cm}$ rectangular opening in the chamber wall. Located $0.2 \mathrm{~cm}$ behind the response key was a Magnavox (Model CK3923) color television. This was connected to a computer programmed to generate and display visual stimuli. A black opaque strip blocked the top half of the screen from view in four of the boxes and the bottom half of the screen in the other four boxes. The visible portion of the screen was conceptually divided into thirds along the horizontal dimension. Two stimuli, a purple vertical strip ( $5 \mathrm{~mm}$ wide and $23 \mathrm{~mm}$ tall) and a green equilateral triangle ( $23 \mathrm{~mm}$ on a side), could be presented centered in the leftmost third of the screen. Two other stimuli, a blue circle $(20 \mathrm{~mm}$ in diameter) and a red horizontal strip (23 mm wide and $5 \mathrm{~mm}$ tall), could be presented centered in the middle third of the screen.

The other three walls and ceiling of the chambers were composed of clear Plexiglas. These chambers were placed in soundand light-attenuating shells, with ventilation fans providing background noise of $62-\mathrm{dB}$ re $20 \mu \mathrm{N} / \mathrm{m}^{2}$. On the rear wall of those shells was mounted a speaker that permitted the presentation of an $80-\mathrm{dB}$ white noise. Also on that wall was a $6-\mathrm{W}$ bulb that was continuously illuminated during the session, except during the operation of the food hopper. That hopper contained Purina pigeon grain. The illumination of the bulb could also be interrupted at a rate of $1 / \mathrm{sec}$ to provide a diffuse visual stimulus.

Experimental events were automatically controlled by relay equipment and microprocessors located in an adjoining room.

Procedure. Because of the birds' past experience in an autoshaping experiment, no magazine training was necessary. However, to ensure low initial rates of responding to the stimuli, the experiment began with 6 days of preexposure to the four colored forms. On each day, the animals received 125 -sec presentations of each stimulus, with a variable intertrial interval (ITI) having a mean of $1 \mathrm{~min}$. On these days, no grain presentations occurred.

On each of the next 8 days, the birds received discrimination training between the vertical strip and the triangle $(\mathrm{A}+/ \mathrm{B}-)$. Half of the animals received reinforced presentations of vertical strip and nonreinforced presentation of the triangle. The other half of the animals had the reinforcement contingencies interchanged. Each animal also received discrimination between the circle and the horizontal strip $(\mathrm{C}+/ \mathrm{D}-)$. In a manner counterbalanced with regard to the $\mathrm{A} / \mathrm{B}$ training, half of the animals received reinforced presentations of the circle and nonreinforced presentations of the horizontal strip; half had the reversed contingencies. Each stimulus was presented 12 times for $5 \mathrm{sec}$ and, where appropriate, terminated in $5 \mathrm{sec}$ of the availability of grain.

On the next day, each animal received a full discrimination training session. Midway through that session and again at the end, the animals received 1 nonreinforced presentation each of $\mathrm{C}$, 
D, AC, AD, BC, and BD. The order of stimulus presentation was arranged so that each compound appeared equally often in Positions $1,3,4$, and 6 , whereas the elements appeared equally often in Positions 2 and 5. The compounds consisted of the simultaneous illumination of both elements for $5 \mathrm{sec}$.

\section{Results and Discussion}

Discrimination training proceeded rapidly. On the final day of training, the mean rates of responding were 145.9 and 2.4 for the $\mathrm{A} / \mathrm{B}$ discrimination and 114.4 and .3 for the C/D discrimination. Every animal successfully learned both discriminations.

Figure 1 shows the results of the summation test, during which the reinforced $C$ and nonreinforced $D$ were each presented separately and in compound with the reinforced $\mathrm{A}$ and nonreinforced $\mathrm{B}$. To the left are shown the results for $\mathrm{C}$ alone and in compound; to the right are the comparable results for $\mathrm{D}$. It is clear that $\mathrm{C}$ continued to evoke substantial responding during the test, but that level of responding was relatively unaffected by its being accompanied either by the nonreinforced $B$ or by the reinforced $\mathrm{A}$. Responding to $\mathrm{AC}$ was not reliably different from that either to $C$ alone [Wilcoxon $T(13)=61$ ] or to $\mathrm{BC}[T(13)=47.5]$. Responding to $\mathrm{D}$ when it was presented alone or in the company of $B$ was essentially at zero; however, the AD compound evoked substantial responding. A and B were not separately presented during the two test segments; however, during the test session as a whole, A and B continued to produce responding like that observed during discrimination training (169.1 and 0 responses per minute, respectively).

These data show no evidence for summation. Responding to the $\mathrm{AC}$ compound was virtually identical to that to $\mathrm{C}$ alone. Moreover, responding to that compound was actually less than that to A alone $[T(15)=24.5, p<$ $.05]$. Responding to the $\mathrm{AD}$ compound was, of course, greater than that to $\mathrm{D}$ alone, but it was not different from that to A alone. Thus, in no case was responding to a compound greater than that to its excitatory elements.

One obvious reason for failing to observe summation is that responding to the elements might be at a behav-

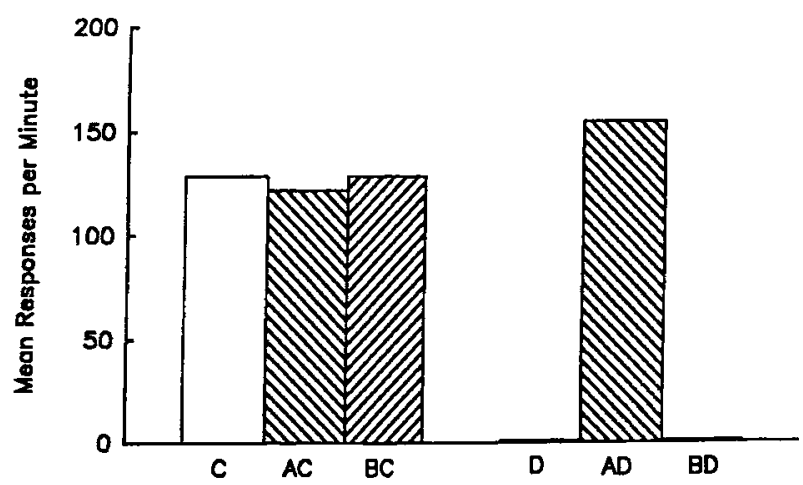

Figure 1. Summation test in Experiment 1. Mean responses per minute during $C$ and $D$, each presented alone and in combination with the previously reinforced $A$ and nonreinforced $B$. ioral ceiling. Although the associative strength of the $\mathrm{AC}$ compound might exceed that of the $\mathrm{A}$ and $\mathrm{C}$ elements, performance limitations might prevent the compound from exhibiting that fact. This seems unlikely because responding to the $\mathrm{AC}$ compound was actually lower than that to the A stimulus alone. Nevertheless, Experiment 2 was explicitly directed at this question.

\section{EXPERIMENT 2}

The aim of Experiment 2 was to look for summation by compounding stimulus elements that individually evoked responding well below a behavioral ceiling. In order to achieve stable intermediate rates of responding to some stimuli, each animal was trained with two sequential compounds, $\mathrm{A}-\mathrm{B}$ and $\mathrm{C}-\mathrm{D}$, each of which terminated in reinforcement. Several prior results suggest that, under these circumstances, $\mathrm{B}$ and $\mathrm{D}$ will control substantial responding whereas $A$ and $C$ will yield only moderate response rates (e.g., Rescorla, 1982; Ricci, 1973). One may then examine summation not only by presenting together the highly excitatory B and D stimuli but also by presenting together the moderately excitatory $\mathrm{A}$ and $\mathrm{C}$ stimuli.

\section{Method}

Subjects and Apparatus. The subjects were 16 female Carneau pigeons with histories like those in Experiment 1. They had not been exposed to the stimuli used in this experiment, and their treatments in this experiment were random with regard to their histories. The apparatus was identical to that of Experiment 1 , with the exception that the vertical strip was yellow instead of purple.

Procedure. Initially, the animals were preexposed to the stimuli. On each of 2 days, the animals received 12 nonreinforced 5-sec exposures of each element, with an ITI variable around a mean of $1 \mathrm{~min}$. On each of the next 5 days, the animals received conditioning of two sequential compounds, A-B and C-D. Each compound consisted of $5 \mathrm{sec}$ of presentation of one stimulus, followed immediately by $5 \mathrm{sec}$ of another stimulus, followed immediately by $5 \mathrm{sec}$ of grain. Each compound was presented 16 times on each day in quasi-random order, with a mean ITI of $1 \mathrm{~min}$. The stimuli were fully counterbalanced across animals, with the constraint that $A$ and $C$ appeared at different key locations from one another, as did $\mathrm{B}$ and $\mathrm{D}$, so that the $\mathrm{AC}$ and $\mathrm{BD}$ compounds could subsequently be tested in simultaneous presentation.

On the next 2 days, the animals continued to receive these training trials but, in addition, short test sequences were interposed at the midpoint and again at the end of the session. These test sequences each consisted of 1 nonreinforced presentation each of $A$, $\mathrm{C}, \mathrm{AC}, \mathrm{B}, \mathrm{D}$, and $\mathrm{BD}$, presented in balanced order. On the first of these 2 days, the training trials continued to be followed by $5 \mathrm{sec}$ of grain; on the second, grain was omitted from all trials. The intention of the first test was to examine summation for the $\mathrm{A} / \mathrm{C}$ and $\mathrm{B} / \mathrm{D}$ elements, which had different levels of performance. The intention of the second test was to examine summation after performance had been reduced to both sets of stimuli as a result of extinction.

\section{Results and Discussion}

The initial compound training was successful in establishing different levels of performance to the $\mathrm{A} / \mathrm{C}$ and 


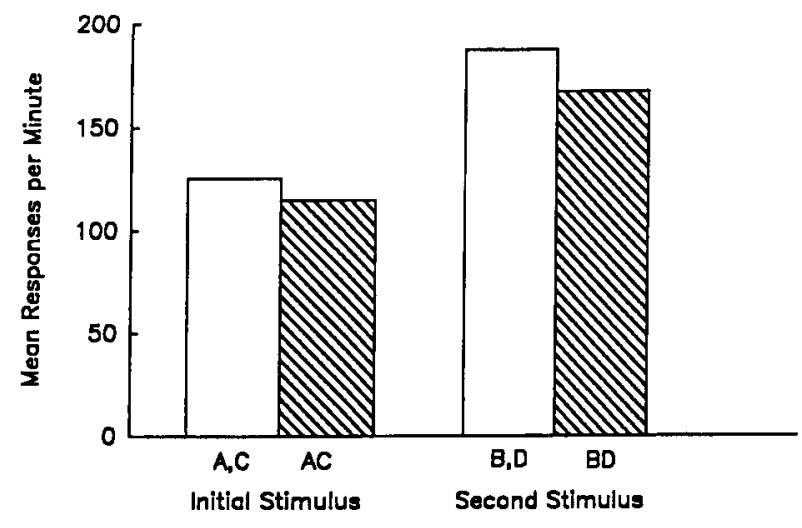

Figure 2. Summation test in Experiment 2. Conditioning consisted of sequential A-B-reinforcer and B-D-reinforcer trials. Responding is shown to the first elements in the conditioning series, $A$ and $C$, and to their compound, as well as to the second elements in the series, B and $D$, and to their compound.

$B / D$ elements. On the final day of training, the mean responses per minute were 85.0 and 145.2 for the leading $\mathrm{A} / \mathrm{C}$ elements and the following $\mathrm{B} / \mathrm{D}$ elements, respectively $[T(15)=9, p<.01]$.

Figure 2 shows the results of the first test session. To the left are the performances to the elements and compound for the first stimulus in the sequence ( $\mathrm{A}$ and $\mathrm{C}$ ); to the right are the corresponding performances for the second stimulus in the sequence (B and D). During the test, the $B / D$ stimuli continued to elicit greater responding than did the $\mathrm{A} / \mathrm{C}$ stimuli $[T(15)=12, p<.01]$. However, neither set of stimuli showed any evidence of greater responding to the compound than to the elements $[T \mathrm{~s}(15)>54]$.

Over the course of the extinction session, responding deteriorated to all of the stimuli. On the test trials, the mean rates of responding were 32.6 and 31.2 to the elements and compound of the first stimulus in the sequence; they were 70.8 and 75.0 to the elements and compound of the second stimuli in the sequence. Responding to the $\mathrm{B} / \mathrm{D}$ elements continued to be greater than that to the $\mathrm{A} / \mathrm{C}$ elements $[T(13)=10, p<.01]$, but responding to neither compound exceeded that to its elements $[T \mathrm{~s}(14)>38]$.

These results show no evidence of summation as a result of presenting a stimulus compound. Element performance was observed at various response levels, but at none did stimulus combinations produce augmented responding. Note particularly that the failure to observe summation with the $\mathrm{A} / \mathrm{C}$ elements occurred at times when the birds did demonstrate substantially greater responding to the $B / D$ elements and compounds. These data suggest that the failure to see summation is unlikely to be the result of failure to take the measurements at an appropriate level of responding.

\section{EXPERIMENT 3}

There are several ways, other than ceiling effects, in which performance might fail to reflect actual summation of associative strength. Experiment 3 explored two of those ways, both stemming from the fact that in prior experiments the conditioned stimuli were presented adjacent to each other in the compound test. This mode of stimulus presentation may introduce complexities in interpreting response rate as an index of overall associative strength. First, it may encourage competing response tendencies that could mask actual summation. During training, $\mathrm{A}$ and $\mathrm{C}$ both elicited considerable responding, but this responding was presumably directed at each of them individually. When they are presented side by side in the AC compound, it seems quite likely that each tends to elicit pecking at its location. This introduces the possibility that there is competition for the location of the peck response that reduces the overall peck rate. Hence, in Experiment 3, competition was reduced by employing compounds in which the elements could be superimposed at the same location.

Second, the summation test in Experiments 1 and 2 compared responding evoked by the $\mathrm{A}$ and $\mathrm{C}$ elements with that evoked by the $\mathrm{AC}$ compound. But these stimuli differ in their spatial extension and thus potentially in the degree to which they might successfully evoke responding, aside from any differences in associative strength. Informal observations in our laboratory have suggested that smaller stimuli evoke higher peck rates than do larger stimuli, even when they have had similar training histories. Hence, the comparison between responding to $\mathrm{AC}$ and that to $\mathrm{BC}$ in Experiment 1 may be the more appropriate data from which to evaluate summation. Those stimuli share their spatial extent but presumably differ in their total associative strength. Nevertheless they showed no evidence of summation. Experiment 3 included a related comparison. In this experiment, animals received pairings of food with $\mathrm{A}$ and $\mathrm{B}$, as well as with a $\mathrm{CD}$ compound. They were then tested for their responding to $\mathrm{AB}$ and $\mathrm{CD}$. Most theories of conditioning would suppose that training of the $C D$ compound would result in overshadowing of $C$ and $D$, such that the total associative strength of $C D$ would be less than that of A plus B. Hence, with counterbalancing of the identities of the stimuli, one can compare responding to the perceptually comparable $\mathrm{AB}$ and $\mathrm{CD}$ compounds when the associative strengths of their elements are different and hence summation should be observable.

In addition, the test procedure used in Experiment 3 differed from that of Experiments 1 and 2 in that all trials were reinforced. Although Experiments 1 and 2 involved relatively few compound test presentations, their nonreinforcement raises the possibility that rapid extinction might have masked summation.

\section{Method}

Subjects and Apparatus. The subjects were 16 female Carneau pigeons with histories like those in Experiment 1. They had not been exposed to the stimuli used in this experiment, and their treatments in this experiment were random with regard to their histories. The apparatus was identical to that of Experiment 1 , with the exception that different stimuli were used. In this experiment, $\mathrm{A}$ and $\mathrm{C}$ were the horizontal green strip used in Experiment 1 and a red vertical strip identical in shape to the purple 
strip used in Experiment 1. The B and D stimuli were circles of the same extent as the red circle used in Experiment 1. One was yellow with superimposed 1 -mm-wide black stripes spaced $2 \mathrm{~mm}$ apart and oriented $45^{\circ}$ from the vertical; the other was blue, with superimposed 1-mm-wide black stripes spaced $1 \mathrm{~mm}$ apart and oriented $135^{\circ}$ from the vertical. When the compounds were presented, the red and green strips were superimposed onto the circles with the same centers.

Procedure. Initially, the animals received 2 days of preexposure to the stimuli. On each day, they received 125 -sec nonreinforced presentations of each of the stimuli, spaced with a mean ITI of $1 \mathrm{~min}$.

On each of the following 4 days, the animals received reinforced training of two elements and one compound. Each trial involved the 5-sec presentation of a stimulus, terminating in $5 \mathrm{sec}$ of grain availability. There were 12 trials with each of the elements and with the compound. For half of the animals, the elements were the red strip and the blue circle whereas the compound consisted of the green strip and yellow circle. For the other half of the animals, the red strip and blue circle were presented in compound whereas the green strip and yellow circle were presented as elements.

On the next day, the animals received a conditioning session followed without interruption by a test sequence. This sequence consisted of 4 reinforced presentations of each of the training elements and training compound, as well as 4 reinforced presentations of the compound composed of the joint occurrence of the training elements. The order of stimulus presentation was balanced across animals.

\section{Results and Discussion}

Conditioning was rapid to the elements and the compound. On the final day, the mean response rates were 84.0 and 92.6 responses per minute, respectively. Figure 3 shows the results of the test session. Responding is displayed separately for the trained elements and trained compound as well as for the compound composed of those trained elements. It is clear that all three types of stimuli evoked similar response rates. The level of responding to the compound of the trained elements was not different from either that to the trained compound or that to the elements themselves $[T \mathrm{~s}(16)>76]$.

There was no evidence that responding to the compounds or the elements changed over the course of the testing. On the second block of two test trials, the mean

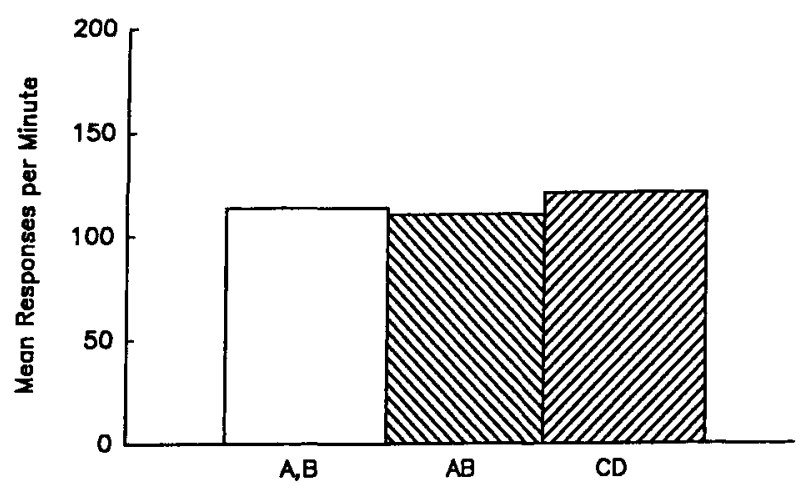

Figure 3. Summation test in Experiment 3. Mean responses per minute during the trained $A$ and $B$ elements and their compound, as well as to the trained CD compound. responses per minute were $115.3,111.4$, and 120.0 for the elements, the compound of those elements, and the trained compound, respectively.

No evidence of summation was found in Experiment 3 , despite the fact that the test compound could be compared to a comparable trained compound and despite the fact that the compound consisted of elements superimposed on one another. This result reduces the plausibility of a competing-response notion. This forces us to entertain more seriously the possibility that the associative strength of the compound does not consist of the combination of the strengths of the elements.

\section{EXPERIMENT 4A}

The purpose of Experiment 4A was to assess further the possibility that performance factors prevent the exhibition of successful summation of the associative strengths of two elements in a compound. It used as the measure of associative strength not responding to the compound but the ability of that compound to produce second-order conditioning of another stimulus. For this purpose; birds received autoshaping with two stimuli, A and $B$. Three other stimuli were then given second-order conditioning in which one stimulus signaled $\mathrm{A}$, one signaled $B$, and one signaled the $A B$ compound. Secondorder conditioning is readily obtained in this preparation, and it sometimes yields an especially sensitive indication of the associative strength of a stimulus (e.g., Rescorla, 1980). If the $\mathrm{AB}$ compound has the summed associative strength of $A$ and $B$, then even if that summation does not show in enhanced responding to $A B$, it might be evident in the enhanced ability of $A B$ to second-order condition its signal. In particular, a compound that had high associative strength might successfully yield enhanced second-order conditioning even though it failed to show that strength in responding because of competing responses, of having a greater spatial distribution, or of a performance ceiling.

\section{Method}

Subjects and Apparatus. The subjects were 16 female Carneau pigeons with histories like those in Experiment 1. They had not been exposed to the stimuli used in this experiment, and their treatments in this experiment were random with regard to their histories. The apparatus was identical to that of Experiment 1, with the exception that different stimuli were used. Two stimuli, a purple diamond measuring $12 \mathrm{~mm}$ on a side and a yellow square measuring $18 \mathrm{~mm}$ on a side, served as the first-order stimuli. The diamond appeared in the left third of the screen and the square on the right third. Three white stimuli, appearing in the center of the screen, served as the second-order stimuli. These were a fivepointed star whose points lay on the imaginary circumference of a circle $24 \mathrm{~mm}$ in diameter, a "target" composed of a set of black and white concentric circles each $1 \mathrm{~mm}$ thick with an outer diameter of $20 \mathrm{~mm}$, and a white $X$ composed of two $5-\mathrm{mm}$ white lines each $23 \mathrm{~mm}$ long crossing in the center of the screen.

Procedure. Initially, all animals received first-order conditioning of the diamond and square. On each of 8 days, they received 165 -sec presentations of each stimulus, terminating in $5 \mathrm{sec}$ of grain, with a mean ITI of $1 \mathrm{~min}$. 
On each of the next 3 days, nonreinforced presentations of the eventual second-order stimuli were intermixed with continued first-order conditioning. Each day contained 16 reinforced presentations of the diamond and square as well as 12 nonreinforced 5-sec presentations each of the star, target, and X. The intention of this exposure was to guarantee low levels of responding to the second-order stimuli prior to their conditioning.

On the next 3 days, all animals received second-order conditioning of the star, target, and X, using the first-order stimuli as reinforcers. Each session contained six 5-sec presentations of each second-order stimulus, terminating in a $5-\mathrm{sec}$ presentation of the diamond, the square, or the diamond/square compound. The assignment of which second-order stimulus signaled the first-order elements and compound was balanced across animals. No deliveries of grain occurred on second-order conditioning trials. However, in order to maintain the associative strength of the diamond and square, all animals received four separate reinforced presentations of those stimuli (cf. Rescorla, 1980). Throughout these sessions, the ITI was variable around a mean of $2 \mathrm{~min}$.

\section{Results and Discussion}

First-order conditioning proceeded smoothly. On the final day of conditioning, the mean responses per minute to the first-order stimuli was 177.8. On that day, the mean responses per minute to the preexposed secondorder stimuli was 3.2 .

Figure 4 shows the course of second-order conditioning, displaying separately the rates of responding for the stimuli that signaled the square or diamond element and those that signaled the square/diamond compound. Over the course of conditioning, the response rate increased to both types of second-order stimuli. However, there was no indication of summation as measured by greater responding to the stimulus signaling the compound. In fact, by the 3rd day of conditioning, the average responding to the two stimuli signaling the elements was greater than that to the stimulus signaling the compound $[T(14)=15, p<.05]$.

On the second-order conditioning trials, one can obtain a measure of pecking not only at the second-order stimulus but also at the subsequent first-order stimulus. On the 3 second-order conditioning days, the mean responses per minute during the first-order elements were

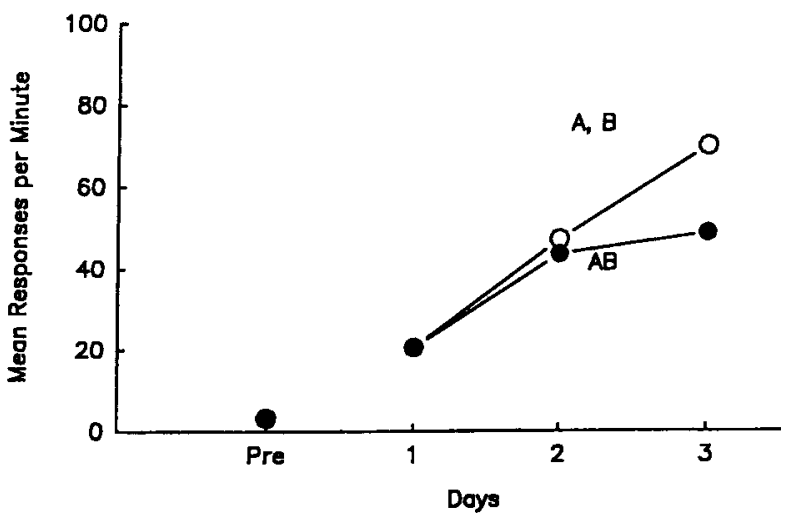

Figure 4. Second-order conditioning in Experiment $4 A$ of localized keylights by previously conditioned $A$ and $B$ elements, and by their compound. The $A$ and $B$ elements were localized keylights.
$177.3,184.3$, and 230.3; the comparable response rates during the compound of these elements were 136.4, 156.1, and 162.1. On none of the 3 second-order conditioning days was there any evidence of greater responding during the compound than during the elements. This observation is of particular interest since the summation tests of the preceding experiments have typically involved only a few presentations. That allows the possibility that evidence of summation might have emerged with repeated testing, perhaps because of reduced disruptive effects of stimulus novelty. Over the 3 days as a whole, the superior responding to the elements proved highly reliable $[T(16)=3, p<.01]$. Just as the elements produced greater conditioning of preceding stimuli, they also evoked greater responding during their own presentation.

\section{EXPERIMENT 4B}

The results of Experiment 4A strongly suggest that the failure to obtain summation in the combination of two keylights signaling food represents actual failure to summate the associative strengths, rather than some performance artifact. One possibility is that two visually localized keylights interact perceptually in such a way that the compound fails to preserve the integrity of each of the elements. It is worth noting that in many successful instances of summation in other preparations, the elements were chosen from different sensory modalities. Indeed, Kehoe, Horne, Horne, and Macrae (1994) recently reported greater summation using mixed modality compounds compared with same modality compounds in eyelid conditioning with the rabbit. It therefore is of interest to ask whether one could obtain summation in the autoshaping preparation if the compound was composed of elements from different modalities. Consequently, in Experiment 4B, summation was assessed for elements more like those used in other preparations, diffuse auditory and visual stimuli.

Of course, pigeons do not direct pecking at such diffuse stimuli when they signal food. As a result, conditioning cannot be assessed in terms of directed pecking at the stimuli. However, when diffuse auditory and visual stimuli signal food, they do take on the ability to produce pecking at localized keylights when they reinforce those keylights in a second-order conditioning procedure (e.g., Nairne \& Rescorla, 1981). Consequently, in Experiment 4B, two diffuse stimuli were first paired with food and then used as elements or as a compound to second-order condition keylights.

\footnotetext{
Method

Subjects and Apparatus. The subjects were like those of the previous experiments. The apparatus was the same as that of Experiment 1.

Procedure. Except for the identity of the stimuli, the procedure was like that of Experiment 4A. The animals first received 8 days of first-order conditioning of a diffuse white noise and a $1 / \mathrm{sec}$ interruption of the houselight. Each stimulus was presented for $5 \mathrm{sec}$
} 


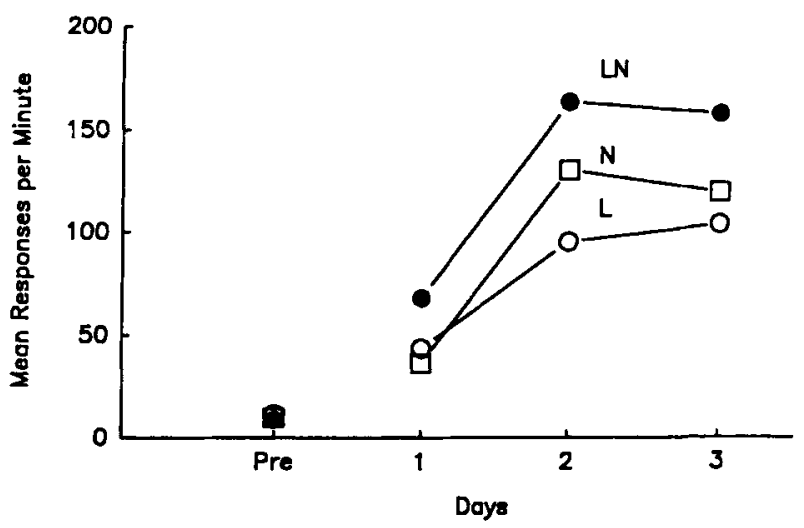

Figure 5. Second-order conditioning in Experiment 4B of localized keylights by previousty conditioned noise (N) and light (L) and by their compound.

and terminated in $5 \mathrm{sec}$ of grain. On the next 3 days, this conditioning continued, but 12 nonreinforced presentations were also given of each of the keylights that would be given second-order conditioning. These stimuli were the target, presented in the center third of the screen, a yellow square, presented in the right third of the screen, and a green triangle presented in the left third of the screen.

On each of the next 3 days, the birds received second-order conditioning of the keylights using the light, the noise, or the light/ noise compound as the reinforcer. Each session contained six 5 -sec presentations of each keylight, terminating in $5 \mathrm{sec}$ of either one of the elements or the compound. In addition, each animal received four reinforced 5 -sec presentations of the light and the noise presented separately. The ITI was variable around a mean of $2 \mathrm{~min}$.

\section{Results and Discussion}

As expected, little pecking occurred during the firstorder conditioning of the noise and light. Figure 5 shows the mean responses per minute to the keylights on the final day of preexposure and over the course of secondorder conditioning. The results are shown separately for the keylights that signaled the light, the noise, and the compound. Although initially there was little responding to the keylights, with the institution of second-order conditioning, responding rapidly increased for all three stimuli. However, the finding of primary interest is the superiority of responding to the stimulus that signaled the noise/light compound. Over the 3 days of conditioning, the keylight signaling the compound showed a higher peck rate than did those signaling either of the elements $[T \mathrm{~s}(16)<15, p s<.01]$. Although the noise was numerically more successful in producing conditioning than was the light, this difference did not prove to be reliable.

These results provide the first evidence for summation in the present series of experiments. When diffuse stimuli in different modalities were separately trained, their subsequent combination apparently yielded higher associative strength. As measured by the ability to produce second-order conditioning, this summation agrees with the summation results typically found in other conditioning paradigms.

\section{EXPERIMENT 4C}

Two localized visual stimuli failed to yield summation in Experiment 4A, but two diffuse stimuli from different modalities succeeded in Experiment 4B. The purpose of Experiment $4 \mathrm{C}$ was to seek summation from one localized visual stimulus and one diffuse auditory stimulus. If the difficulty with localized visual stimuli is that they interact perceptually, then one such stimulus should summate with a diffuse auditory stimulus. On the other hand, if there is something inherent in localized visual stimuli that prevents them from showing summation, then summation might fail even when a localized visual stimulus is combined with a diffuse stimulus in another modality. To assess these possibilities, Experiment $4 \mathrm{C}$ was a replication of Experiments $4 \mathrm{~A}$ and $4 \mathrm{~B}$ except for the choice of stimuli.

\section{Method}

Subjects and Apparatus. The subjects were like those of the previous experiments. The apparatus was the same as that of Experiment 1.

Procedure. Except as noted, this experiment was identical to Experiments $4 \mathrm{~A}$ and $4 \mathrm{~B}$. The first-order stimuli were the noise of Experiment $4 \mathrm{~B}$ and a pinwheel (A) composed of 8 black and 8 white sectors composing a circle of $20 \mathrm{~mm}$ in diameter. The second-order stimuli were a black and blue version of the target stimulus used in Experiment $4 B$, a red $X$ identical in dimensions to that used in Experiment $4 \mathrm{~B}$, and a green triangle of the same dimensions as that used in Experiment $4 B$. After 8 days of firstorder conditioning of the noise and target and 3 days of conditioning combined with preexposure to the second-order stimuli, second-order conditioning was conducted over the course of 3 days. It is the rates of responding to the second-order stimuli signaling the noise, the target, and the noise/target compound that constitute the data of interest.

\section{Results and Discussion}

On the final day of preexposure, the mean responses per minute were $151.4,4.0$, and 7.2 during the pinwheel (A), the noise, and the second-order stimuli, respectively.

Figure 6 shows the mean rates of responding to the second-order stimuli at the end of preexposure and over the course of conditioning. This figure shows that all three second-order conditioning stimuli rapidly acquired responding. However, over the 3 days of second-order conditioning, there was greater responding to the keylight signaling the target/noise compound than to either of those signaling the elements $[T \mathrm{~s}(15)<22, p \mathrm{~s}<.05]$.

As in Experiment $4 \mathrm{~A}$, it is possible to examine the response rates during second-order trials to the first-order keylight stimulus when it was the only consequence and when it was presented in compound with the noise. Not surprisingly, on the basis of other evidence (e.g., Rescorla, 1986), the excitatory noise did not enhance responding to the visually localized target stimulus. Over the 3 days of second-order conditioning, the mean responses per minute were 158.3 and 151.1 to the target and the target/noise compound, respectively.

The results of Experiment $4 \mathrm{C}$ thus show that a diffuse auditory stimulus will summate its associative strength 


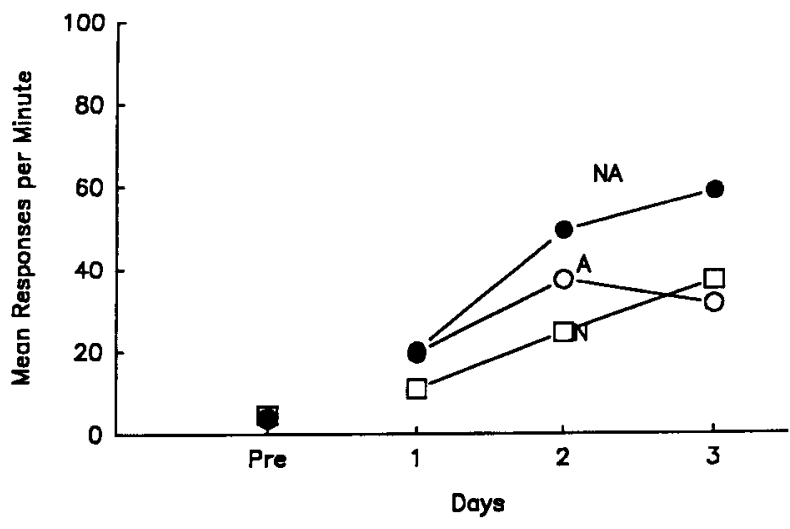

Figure 6. Second-order conditioning in Experiment $4 \mathrm{C}$ of localized keylights by previously conditioned noise $(\mathrm{N})$ and localized keylight (A) and by their compound.

with a localized keylight, when that summation is measured in second-order conditioning. This result is consistent with the view that previous failures of summation with two localized visual stimuli are attributable to their perceptual interaction.

\section{EXPERIMENT 4D}

The results of Experiments $4 \mathrm{~A}-4 \mathrm{C}$ suggest that summation of associative strength fails to occur with compounds of two localized visual stimuli but succeeds with compounds of auditory and visual stimuli. However, these experiments differed from each other in various details, including the identities of the stimuli used as signals of those compounds. For that reason, it seems valuable to directly compare compounds composed of different elements within the same experiment, using the same second-order stimuli.

Consequently, in Experiment 4D, the pigeons first received conditioning of two auditory stimuli and two localized visual stimuli. For each bird, different secondorder stimuli then signaled different compounds composed of two visual elements, two auditory elements, or an auditory and a visual element. Of interest is whether we can confirm, within one experiment, the superior second-order conditioning from a compound composed of an auditory and visual element compared with one composed of two visual elements.

\section{Method}

Subjects and Apparatus. The subjects were like those of the previous experiments. The apparatus was the same as that of Experiment 1 .

Procedure. This experiment followed the general procedures of the Experiments $4 \mathrm{~A}-4 \mathrm{C}$. The birds first received 5 days of preexposure to the second-order stimuli, in order to ensure low levels of responding prior to pairing with the compounds. On each day, they received 12 nonreinforced 5 -sec presentations of each of four stimuli: the red vertical and green horizontal strips used in Experiment 3, as well as two other strips of the same dimensions, one yellow and slanted $45^{\circ}$ from the vertical and one blue and slanted $135^{\circ}$ from the vertical.

On each of the next 6 days, the birds received first-order conditioning. On each day, they received 125 -sec reinforced presentations each of the white noise, an $1800-\mathrm{Hz}$ tone of the same intensity, a brown diamond of the same dimensions used in Experiment $4 \mathrm{~A}$, and a striped circle identical to that used in Experiment 3 except that black lines appeared on a white, rather than a yellow, background. All stimuli appeared in the center of the key.

On each of the next 4 days, the animals continued to receive first-order conditioning, but with nonreinforced exposures of the eventual second-order stimuli intermixed. On each day, they received 6 reinforced presentations each of the four first-order stimuli and 12 nonreinforced presentations each of the four secondorder stimuli.

On the next 3 days, the birds received second-order conditioning. Each session contained 6 presentations of each second-order stimulus followed by a compound of two first-order stimuli. In addition, there were 3 reinforced presentations of each of the firstorder elements. The four compounds used were composed of two keylights (the diamond superimposed on the striped circle), two auditory stimuli (noise and tone), or an auditory and visual stimulus (noise/diamond and tone/circle for half of the animals and noise/circle and tone/diamond for the other half of the animals). The assignment of second-order stimulus to compound was fully counterbalanced across animals. Throughout this phase, the ITI was variable around a mean of $2 \mathrm{~min}$.

\section{Results and Discussion}

First-order conditioning and pretesting proceeded without incident. On the day before the beginning of second-order conditioning, the mean responses per minute to the first-order keylights and the second-order stimuli were 168 and 2.5 , respectively; responding seldom occurred during the auditory signals of food.

Figure 7 shows responding to the second-order stimuli over the course of conditioning, displaying separately the responding to the second-order stimuli followed by the purely visual compound, the purely auditory compound, as well as the average of the two second-order stimuli that signaled the auditory/visual compounds. It is clear that the purely visual compound was less successful in producing second-order conditioning than

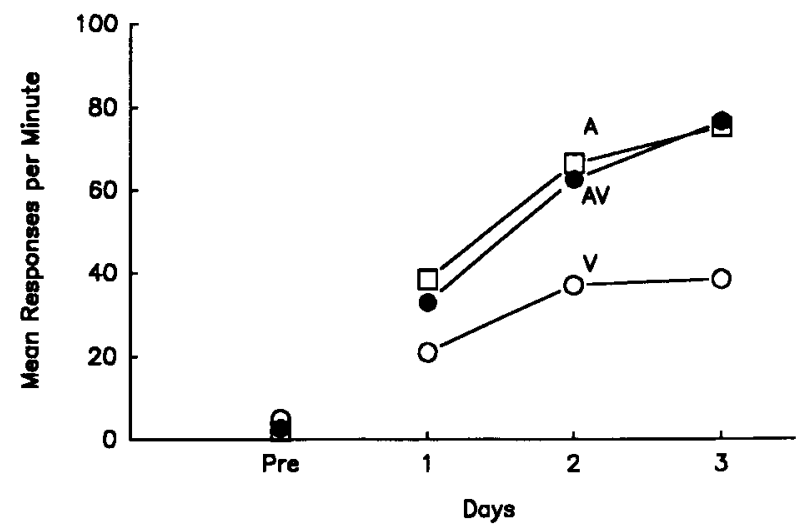

Figure 7. Second-order conditioning in Experiment 4D of localized keylights by compounds of two visual elements (V), two auditory elements (A), or an auditory and visual element (AV). 
were the other two. Over the 3 days of conditioning, responding to the signal of the visual compound was reliably less than that to any of the other signals $[\mathrm{Ts}(14)<$ $17, p<.05]$.

These results agree with those of the previous experiments in suggesting that associative strengths combine more successfully for an auditory and a visual stimulus than for two visual stimuli. Of course, the design of Experiment $4 \mathrm{D}$ precludes comparison with signals of the first-order elements themselves. Consequently, on the basis of this experiment alone, little can be concluded about summation per se. But, when taken in conjunction with the results of Experiments $4 \mathrm{~A}-4 \mathrm{C}$, these results suggest that perceptual interactions among visual stimuli may prevent them from showing summation when presented in combination. The results of Experiment 4D also suggest the possibility that such interactions are absent from auditory stimuli, but other designs would clearly be needed to determine whether summation can be directly observed in that case.

If the failure to observe summation with localized visual stimuli results from their perceptual interaction, then procedures that manipulate that interaction should affect the amount of summation. The purpose of Experiment 5 was to take a theoretical approach to such a manipulation, whereas Experiment 6 was more empirically motivated.

\section{EXPERIMENT 5}

One attractive account of the failure to achieve summation with localized visual stimuli is that the compound is processed as a configuration, not analytically. But the fact that some responding does occur to the compound after training of its elements implies that the organism does not treat it as an entirely new stimulus, unrelated to those that have been trained. Hence, the challenge for a configural theory is to specify the basis of that generalization from the elements to compound.

The most successful modern specification of this sort is the theory proposed by Pearce (1987). As noted above, under the most simple circumstances, this theory does not anticipate that appreciable summation will occur. However, one may use the theory to develop conditions that should generate summation. One such set of conditions arises if one makes the quite reasonable assumption that two stimulus elements, A and B, have both unique and shared features. Therefore, they might better be represented as $\mathrm{AX}$ and $\mathrm{BX}$, with the compound being represented as ABX. As Pearce (1987) notes, under this set of assumptions, the similarity of each of the AX and BX elements to the ABX compound exceeds .5 and, as a result, the configural model does in fact anticipate a small level of summation. However, the magnitude of that anticipated summation turns out to be quite modest, and it does not vary in any simple way with the relative salience of the shared feature, $X$. For this reason, it is difficult to generate a simple prediction about the amount of summation as a function of the similarity of the elements. Consequently, the contrary finding of greater summation in Experiments $4 \mathrm{~B}$ and $4 \mathrm{C}$ than in Experiment $4 \mathrm{~A}$ is not a strong basis for rejecting the Pearce theory.

However, a much stronger prediction about summation can be made about a situation in which $\mathrm{ABX}$ is tested after training of $\mathrm{AX}$ and $\mathrm{BX}$ which is augmented by separate nonreinforced presentations of $\mathrm{X}$ alone. With such a procedure, $X$ will develop inhibitory strength because it receives excitatory generalization from both $\mathrm{AX}$ and $\mathrm{BX}$ but is nonreinforced. Because this inhibition in turn will generalize back to $\mathrm{AX}$ and $\mathrm{BX}$, these reinforced compounds will become especially excitatory, each compensating for the inhibition. Under these circumstances, the triplet, $\mathrm{ABX}$, is expected to have a greater net associative strength than either $\mathrm{AX}$ or $\mathrm{BX}$ alone. This deduction can be seen intuitively as follows: because $\mathrm{AX}$ and $\mathrm{BX}$ have separately compensated for the inhibition controlled by $\mathrm{X}$, the triplet receives from $\mathrm{AX}$ and $\mathrm{BX}$ twice the generalized excitation it needs to overcome the generalized inhibition it receives from X. More formally, on the assumption that the reinforcer supports an asymptotic strength of 1 , one can show that responding to $\mathrm{ABX}$ should equal $1+I_{X} /\left(I_{X}+\right.$ $I_{\mathrm{A}}+I_{\mathrm{B}}$ ), where $I_{\mathrm{X}}$ represents the salience or intensity of $X$. That is, according to this theory, the more salient is the shared element $X$, the more summation should be observed from combining A and B.

There are some data that seem to question that prediction. For instance, Pearce et al. (1992) tested an ABX stimulus after training of the form $\mathrm{AX}+, \mathrm{BX}+, \mathrm{X}-$. The focus of their experiment was on comparing responding to $A B X$ with that to another triplet, CDY, whose elements had received $\mathrm{C}+, \mathrm{D}+, \mathrm{Y}-$ training. Consequently, they did not directly compare responding to $\mathrm{ABX}$ with that to $\mathrm{AX}$ and $\mathrm{BX}$. However, terminal levels of responding to $\mathrm{AX}$ and $\mathrm{BX}$ were very similar to the reported levels of responding to $A B X$ in the test, suggesting a failure to obtain summation.

The present experiment explored this prediction more directly. Pigeons were first trained in a discrimination of the form $\mathrm{AX}+, \mathrm{BX}+, \mathrm{X}-, \mathrm{C}+, \mathrm{D}+$. Simple summation was then tested by comparing responding during $C D$ with that during $C$ and $D$; summation was also tested by comparing responding to $\mathrm{ABX}$ with that to $\mathrm{AX}$ and $\mathrm{BX}$. On the basis of the results of Experiments 1-3, we would anticipate finding no summation with $\mathrm{CD}$. However, on the basis of the Pearce account of configural processing, we would anticipate considerable summation with $\mathrm{ABX}$.

\section{Method}

Subjects and Apparatus. The subjects were 16 female Carneau pigeons with histories like those in Experiment 1. They had not been exposed to the stimuli used in this experiment, and their treatments in this experiment were random with regard to their histories. The apparatus was identical to that of Experiment 1, with the exception that different stimuli were used. In this experiment, five stimuli were used: a brown vertical strip of the same dimensions as the vertical strip used in Experiment 1, a blue triangle of the same dimensions as the triangle used in Experiment 1, a red 
circle identical to that used in Experiment 1, a green square measuring $18 \mathrm{~mm}$ on a side, and the yellow five-pointed star used in Experiment 4A. The brown strip always occurred in the middle portion of the screen, the triangle and the circle always appeared in the left portion, and the star and the square always appeared in the right portion.

Procedure. On each of 5 conditioning days, the animals received 8 reinforced presentations each of $\mathrm{C}, \mathrm{D}, \mathrm{AX}$, and $\mathrm{BX}$, as well as 16 nonreinforced presentations of $X$. For all animals, the brown strip served as X. For half the animals, A and B were the triangle and the square, and $C$ and $D$ were the circle and the star. For the other half of the animals, the reverse identifications were in effect.

On the next day, the animals received an additional conditioning session, except that two test periods were given, one in the middle of the session and one at the end. Each test period contained 1 nonreinforced presentation each of $\mathrm{AX}, \mathrm{BX}, \mathrm{ABX}, \mathrm{AB}, \mathrm{C}$, $\mathrm{D}, \mathrm{CD}$, and CDX, presented in counterbalanced order.

\section{Results and Discussion}

Initial conditioning was uneventful. By the final day, all animals were responding enthusiastically to the reinforced stimuli, but there were few responses to the nonreinforced $\mathrm{X}$. On this day, the mean responses per minute were $157.8,145.4$, and 1.3 to the $\mathrm{C} / \mathrm{D}, \mathrm{AX} / \mathrm{BX}$, and $\mathrm{X}$ stimuli, respectively.

Figure 8 shows the results of the test periods, separated for the $A X / B X$ and $C / D$ pairs. The results of the testing with $\mathrm{CD}$ and $\mathrm{CDX}$ confirm those of the previous experiments. There was no evidence of summation in the responding to $C D$. In fact, in Experiment 5, responding to the CD compound was reliably below that to $C$ and $D$ separately $[T(15)=24.5, p<.05]$. Responding to CDX was similar to that to $C D$, suggesting that $X$ had little excitatory or inhibitory power on its own.

The results of testing with $\mathrm{ABX}$ (displayed at the right in Figure 8) also show little evidence of summation. Responding remained high to the trained $\mathrm{AX}$ and BX stimuli. But there was no evidence at all of summation in responding to $A B X$. In fact, responding to the $A B X$ compound was reliably lower than that either to the $\mathrm{AX}$ and BX components $[T(15)=9, p<.01]$ or to the compound presentation of $\mathrm{AB}[T(14)=9.5, p<.01]$.

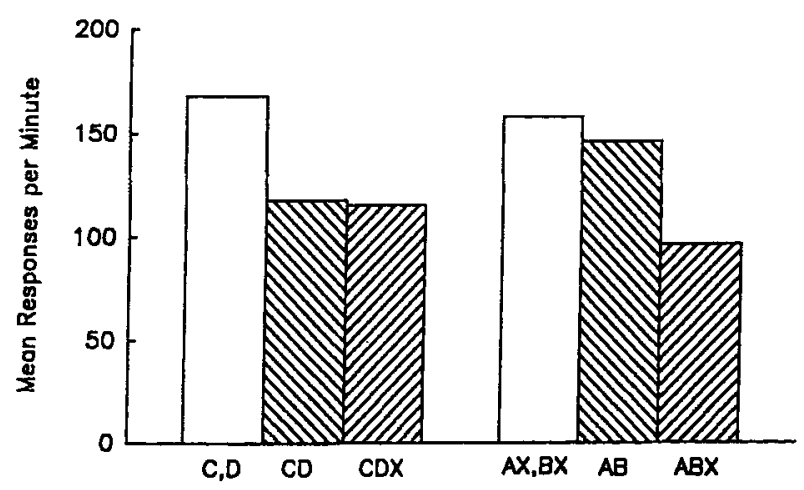

Figure 8. Summation test in Experiment 5. Responding is shown to the previously conditioned $\mathrm{C}, \mathrm{D}, \mathrm{AX}$, and $\mathrm{BX}$ stimuli as well as to various compounds.
The failure to see summation to the CD compound is in agreement with the prediction of the Pearce configural model, although the reliably lower level of responding to this compound compared with its elements is not. More importantly, the failure to see summation with ABX contradicts the prediction of this model. The contradiction is especially powerful because the model anticipates greater responding to $\mathrm{ABX}$ than to $\mathrm{AB}$ alone. The reason for this is that there should be greater similarity between $\mathrm{ABX}$ and the $\mathrm{AX} / \mathrm{BX}$ elements than there is between $A B$ and those elements. Yet these results show greater responding to $\mathrm{AB}$ than to $\mathrm{ABX}$. Thus, these results suggest that if summation fails because the compound stimulus is treated as a configure, the Pearce model fails to accurately capture this configural representation.

It is worth noting that the prediction deduced here from the Pearce model applies not only to discrete stimuli but also to contextual stimuli that might play the role of $\mathrm{X}$. Whenever two discrete stimuli, $\mathrm{A}$ and $\mathrm{B}$, are conditioned, they may be viewed as receiving this conditioning in some context. If this context is labeled $X$, then the training consists of $\mathrm{AX}+, \mathrm{BX}+$, and $\mathrm{X}-$ trials. Hence, this configural model in general anticipates that greater summation will occur between two elements when they are conditioned and tested in a more salient context. Models of this sort can sometimes make qualitatively different predictions when the role of the context is taken into account. However, the present data suggest that such an appeal to context will not readily enable this theory to encompass the summation data for the localized visual stimuli used in autoshaping.

\section{EXPERIMENT 6}

Although the configural theory suggested by Pearce seems not to provide much guidance for reducing the perceptual interaction among localized visual stimuli, other less theoretical considerations might be helpful. In Experiment 6, an attempt was made to reduce the perceptual interaction by separating the stimuli temporally. The idea was that if the elements of a compound were presented sequentially, rather than simultaneously, then perceptual effects of the elements on each other might be reduced. One could then look for summation in terms of responding to an element when it was presented alone or as the second component of a compound. To examine this possibility, the birds used in Experiment 1 were given additional testing with sequential compound presentation.

\footnotetext{
Method

Subjects and Apparatus. The subjects and apparatus were those of Experiment 1.

Procedure. Beginning with the day after their initial simultaneous compound test, the animals were given 3 additional test days with the same structure. That is, midway through a discrimination session and again at the end, they received 1 nonreinforced presentation each of $\mathrm{C}, \mathrm{D}, \mathrm{AC}, \mathrm{AD}, \mathrm{BC}$, and $\mathrm{BD}$, given in balanced order. On the first two of these additional test sessions, the ele-
} 
ments were presented sequentially on the compound tests. The 5 $\sec A$ and $B$ stimuli occurred immediately prior to the 5 -sec $C$ and $D$ stimuli. On the third of these sessions, the compounds consisted of simultaneous presentations of the elements, exactly as in the test session of Experiment 1. Taken together with the test procedure of Experiment 1, these tests yield results of simultaneous and sequential compound testing, given in counterbalanced order.

\section{Results and Discussion}

Figure 9 shows the results of the four test sessions. Plotted are the mean response rates to $C$ when it was presented alone and in compound with the excitatory $A$ and nonreinforced $\mathrm{B}$. To the left in the figure are shown the results of the two simultaneous test sessions. Not surprisingly, there was no evidence of suppression from that simultaneous test. Responding was virtually identical during $\mathrm{C}$ whether it was presented alone or accompanied by either $\mathrm{A}$ or $\mathrm{B}$.

To the right in the figure are shown the results of the two sequential test sessions on which $\mathrm{C}$ was presented alone or preceded by $\mathrm{A}$ or $\mathrm{B}$. These results show evidence of summation. Responding to $C$ was enhanced when it was preceded by the excitatory $A$ compared both with its presentation alone $[T(15)=18, p<.02]$ and with its being preceded by B $[T(14)=14.5, p<.02]$. This result suggests that the differential associative strengths controlled by $\mathrm{A}$ and $\mathrm{B}$ differentially combined with that of $\mathrm{C}$ to yield summation.

The greater level of responding to $\mathrm{C}$ produced by the sequential $\mathrm{A}-\mathrm{C}$ presentation, compared with simultaneous AC presentation, also proved reliable $[T(16)=8$, $p<.01]$. This suggests greater summation in the case of sequential presentation.

An obvious interpretation of this result is that, by separating $A$ and $C$ in time, we encouraged the separate perceptual processing of each stimulus and thus allowed them to retain sufficient integrity to be identified as components of the compound. This suggests that one

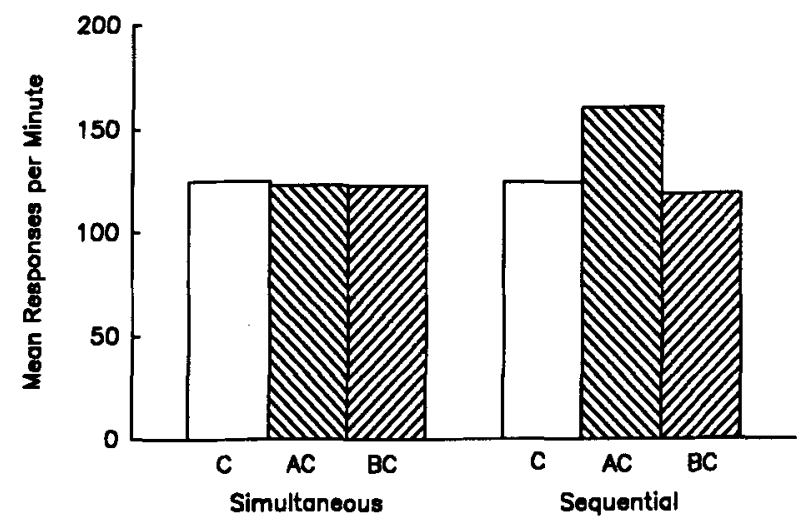

Figure 9. Summation test in Experiment 6. Responding is shown to the conditioned $C$ when presented alone, as well as when compounded with the previously reinforced $A$ and nonreinforced $B$. The results of the $C$ stimulus being preceded by $A$ and $B$ are shown on the left. The results of the simultaneous compound test from Experiment 1 are shown on the right. might be able to use sequential stimulus presentation to detect summation of associative strengths for stimuli that otherwise interact perceptually.

On the other hand, this result is not without alternative interpretations. For instance, one consequence of presenting $\mathrm{A}$ prior to $\mathrm{C}$ is that the pecking elicited by $\mathrm{A}$ will guarantee that the bird is proximal to the key and engaged in pecking when $\mathrm{C}$ appears. This may improve pecking to $\mathrm{C}$ either because it reduces the latency for initiating pecking or because it speeds the processing of $\mathrm{C}$, rather than because it produces the summation of associative strengths. A similar augmentation would not be expected from the nonreinforced B stimulus. Although one cannot rule out such alternatives, the present results are also consistent with the view that sequential presentation reduced perceptual interaction and hence permitted summation.

\section{GENERAL DISCUSSION}

This series of experiments demonstrates that summation can occur in autoshaping if measures are taken to reduce the perceptual interaction among the elements of the compound. In the absence of such steps, summation failed to occur both for adjacent and for superimposed localized visual stimuli. This was true whether the summation was assessed by responding to the compound or by the ability of that compound to produce second-order conditioning of another stimulus. However, when perceptual interactions were avoided, by using stimuli in different modalities or presenting the stimuli sequentially, then summation was observed.

These observations suggest that the localized visual stimuli conventionally used in an autoshaping paradigm may be a poor choice for the investigation of many problems that involve compound stimuli. To the degree that the issues being tested require that stimulus elements preserve their identities in compounds, then localized stimuli would be inappropriate. Moreover, theories that assume such preservation would naturally not fare well when tested with such stimuli.

On the other hand, theories assuming that compounds are separate stimuli, distinct from their elements, could prove to be especially well suited for the localized visual stimuli normally used in autoshaping. Certainly, one theory that makes this assumption (Pearce, 1987) has enjoyed considerable success with compound data collected in the autoshaping setting. However, that theory did not prove to be very helpful in analyzing the present results. Although it correctly anticipates minimal summation for localized stimuli, it fails to anticipate that summation will be much more substantial under the conditions that here found additivity. It does not expect more summation from the more dissimilar diffuse stimuli than from the more similar localized visual stimuli. In addition, like other trial-level models, it is silent on the importance of temporal relations; consequently, it does not provide a natural account of successful summation 
with sequential compound testing. Finally, when that theory was directly tested in Experiment 5, the results proved contrary to its predictions.

The present data are silent on the nature of the perceptual interactions that might prevent summation. Some instances may involve relatively primitive peripheral interactions. For instance, in the case of the distributed visual stimuli used in Experiment 1, the presence of A might reduce the processing of $B$ because responding is directed at A, and, hence, B is actually more peripheral. Other instances may involve less primitive physical constraints. For instance, in the case of the superimposed stimuli used in Experiments 3 and 4D, the presence of A may change the perception of $B$ through processes such as color contrast.

Of course, the actual range of localized stimulus presentation conditions studied here is quite small. However, it does include the two most frequently employed patterns, adjacency and superimposition. Moreover, one other frequently employed procedure, in which the stimuli are composed of color elements that are intermingled in compound presentation, seems especially likely to result in perceptual interactions (e.g., Pearce \& Redhead, 1993). But clearly more attention needs to be given to the nature of these interactions if one is to build a more specific interpretation of the results of compounding such stimuli.

It is worth noting that apparent summation of associative strengths could come about in a variety of ways. The basic observation is that after training $A$ and $B$, then responding to the $\mathrm{AB}$ compound is greater than responding to those elements. Historically, the most popular interpretation has been in terms of additivity of the associations. However, at least two other alternatives need to be evaluated.

One primitive possibility arises because the compound may allow the animal to use the elemental associations more successfully. For instance, when one element has a higher associative strength than the other, then the compound offers each animal the opportunity to respond to the stronger of its stimuli. If the data are evaluated by comparing this responding to responding during the individually presented elements, then compound responding may be greater not because of true summation but rather because the compound always contains the stronger element for each animal. For this reason, the observation in Experiments $4 \mathrm{~B}$ and $4 \mathrm{C}$ of greater second-order conditioning with the compound than with either of the elements is especially informative.

More difficult to evaluate is the possibility that stimuli are processed only some percentage of the time. Responding to the compound could then be greater simply because there is a higher likelihood that at any particular time at least one stimulus is being processed. Although in some sense such an improvement in responding represents summation, one might be reluctant to accept this as an instance of successful combination of associative strengths.
Another mechanism by which responding can be greater to an $\mathrm{AB}$ compound than to the $\mathrm{A}$ and $\mathrm{B}$ elements emerges from studies of modulation (e.g., Holland, 1983). In some conditioning situations, stimulus A takes on the ability to augment or depress responding to stimulus B not because of the additivity of A and B's associative strengths but because $A$ modulates the success with which B uses its association. Although this means of producing greater responding to the compound is of considerable interest in its own right, it does not represent an additive combination of associative strengths. Indeed, within the modulation literature, authors are frequently at some pains to separate modulation from such summation (Holland, 1983; Rescorla, 1986).

In any case, the present results suggest that instances of summation can be observed with localized visual stimuli in autoshaping. But they also caution against the routine use of compounds of localized visual stimuli for studying general issues of Pavlovian conditioning.

\section{REFERENCES}

Bellingham, W. P., Gillette-Bellingham, K., \& Kehoe, E. J. (1985). Summation and configuration in patterning schedules with the rat and rabbit. Animal Learning \& Behavior, 13, 152-164.

Forbes, D. T., \& Holland, P. C. (1985). Spontaneous configuring in conditioned flavor aversion. Joumal of Experimental Psychology: Animal Behavior Processes, 11, 224-240.

Holland, P. C. (1983). "Occasion-setting" in Pavlovian feature positive discriminations. In M. L. Commons, R. J. Herrnstein, \& A. R. Wagner (Eds.), Quantitative analyses of behavior: Vol. 4. Discrimination processes (pp. 183-206). Cambridge, MA: Ballinger.

Hull, C. L. (1943). Principles of behavior. New York: AppletonCentury-Crofts.

KEHOE, E. J. (1986). Summation and configuration in conditioning of the rabbit's nictitating membrane response, Journal of Experimental Psychology: Animal Behavior Processes, 12, 186-195.

KeHOE, E. J., HORNe, A. J., HORNE, P. S., \& MACraE, M. (1994). Summation and configuration between and within sensory modalities in classical conditioning of the rabbit. Animal Learning \& Behavior, 22, 19-26.

MAckintosh, N. J. (1975). A theory of attention: Variations in the associability of stimuli with reinforcement. Psychological Review, 82, 276-298.

NAIRNe, J. S., \& Rescorla, R. A. (1981). Second-order conditioning with diffuse auditory reinforcers in the pigeon. Learning \& Motivation, 12, 65-91.

Pavlov, I. P. (1927). Conditioned reflexes: An investigation of the physiological activity of the cerebral cortex (G. V. Anrep, Ed. and Trans.). London: Oxford University Press.

Pearce, J. M. (1987). A model of stimulus generalization for Pavlovian conditioning. Psychological Review, 84, 61-73.

Pearce, J. M., AdaM, J., Wilson, P. N., \& Darby, R. J. (1992). Effects of discrimination training on responding during a compound conditioned stimulus. Journal of Experimental Psychology: Animal Behavior Processes, 18, 379-386.

PeArce, J. M., \& Hall, G. (1980). A model for Pavlovian learning: Variations in the effectiveness of conditioned but not unconditioned stimuli. Psychological Review, 82, 532-552.

Pearce, J. M., \& Redhead, E. S. (1993). The influence of an irrelevant stimulus on two discriminations. Journal of Experimental Psychology: Animal Behavior Processes, 19, 180-190.

REBERG, D. (1972). Compound tests for excitation in early acquisition and after prolonged extinction of conditioned suppression. Learning \& Motivation, 3, 246-258.

RESCORLA, R. A. (1980). Pavlovian second-order conditioning. Hillsdale, NJ: Erlbaum. 
ResCorla, R. A. (1982). Effect of a stimulus intervening between CS and reinforcer in autoshaping. Journal of Experimental Psychology: Animal Behavior Processes, 8, 131-141.

RESCORLA, R. A. (1986). Facilitation and excitation. Journal of Experimental Psychology: Animal Behavior Processes, 12, 325-332.

RESCORLA, R. A. (1989). Redundant treatments of neutral and excitatory stimuli in autoshaping. Journal of Experimental Psychology: Animal Behavior Processes, 15, 212-223.

RESCORLA, R. A., \& WAGNER, A. R. (1972). A theory of Pavlovian conditioning: Variations in the effectiveness of reinforcement and nonreinforcement. In A. H. Black \& W. F. Prokasy (Eds.), Classical conditioning II: Current research and theory (pp. 64-99). New York: Appleton-Century-Crofts.

RicCI, J. A. (1973). Key pecking under response-independent food presentation after long simple and compound stimuli. Journal of the
Experimental Analysis of Behavior, 19, 509-516.

Rummelhart, D. C., \& McClelland, J. L. (Eds.) (1986). Parallel distributed processing: Vol. 1. Foundations. Cambridge, MA: MIT Press, Bradford Books.

WEISs, S. J. (1972). Stimulus compounding in free-operant and classical conditioning: A review and analysis. Psychological Bulletin, 78, 189-208.

WhitLOW, J. W., \& WAGNER, A. R. (1972). Negative patterning in classical conditioning: Summation of response tendencies to isolable and configural components. Psychonomic Science, 27, 299-301.

(Manuscript received January 21, 1994;

revision accepted for publication September 3, 1994.) 